\title{
Convergence Across Kazakh Regions
}

\author{
Miriam Frey, University of Regensburg \\ Carmen Wieslhuber, University of Regensburg
}

\begin{abstract}
Even though Kazakhstan is one of the most successful transition countries in Central Asia it has been neglected in the literature on regional convergence. This paper fills the gap with an empirical analysis of the convergence process on the regional level using annual gross regional product (GRP) data for the period 1998-2008 for the 16 Kazakh regions. Sigma convergence implies that the dispersion of per capita GRP, measured as the standard deviation of per capita GRP across regions, declines over time. Given the growing variation in per capita GRP over time this phenomenon cannot be found for Kazakhstan. In the neoclassical growth model, under the assumption of similar steady states, the growth rate of per capita GRP is negatively related to the initial level of per capita GRP. Surprisingly, we do not find this relation for the Kazakh regions. The data show that there is no evidence for absolute beta convergence. In contrast, the Kazakh regions even seem to diverge.
\end{abstract}

JEL codes: O40, O53

\section{Introduction}

Studies on regional convergence within or across countries were already done for a broad range of regions. The most popular work is probably the one by Barro et al. (1991), who identified absolute and conditional beta convergence for the US. An example of a former Soviet Union country can be found in Solanko (2008), who looks at regional convergence in Russia. However, to the best of our knowledge, non of them deals with the former Soviet Union countries in Central Asia. The reason might be the insufficient or even missing data on these countries, especially on the regional level.

Kazakhstan is the biggest among the five former Soviet Union countries in Central Asia. As it is a transition and developing country and in addition well endowed with oil, it is of special interest for studies on regional growth and convergence.

There are already several studies which use regional data for Kazakhstan to work on growth and income distribution. In his work on poverty reduction Agrawal (2008) shows that provinces with higher growth rates achieved a faster decline in poverty. O'Hara and Gentile (2009) instead focus on the regional distribution of Kazakhstan's rising GDP during the postSoviet period. Though, there is no information on regional convergence. The objective of this analysis is to close this gap.

The paper is structured as follows. In section 2 the data used for the empirical analysis is described. Section 3 briefly repeats the theoretical concepts of $\sigma$ - and $\beta$-convergence. This theory is empirically tested in section 4 . The paper concludes with the overview of the main results and some ideas for further research.

\section{Data Description}

Per capita personal income is broadly used in the majority of empirical work done on convergence. However, due to the lack of these data on regional level, we use the gross regional product (GRP) per capita for our analysis. This variable measures factor incomes derived from production within a region. In their paper on convergence Barro and Sala-iMartin (1992) stated that the empirical results for personal income are almost equal to those for GRP. The data in US-Dollar at PPP (purchasing power parity) are drawn from the National Human Development Report 2009. It includes data for the 16 Kazakh regions (oblasts) for the period 1990-2008. 
As in 1997 the capital of Kazakhstan was moved from Almaty to Astana, which subsequently was treated as a distinct region, we have a complete data set for the 16 regions only for the period 1997-2008. Another peculiarity of the Kazakh regional data is that in 1997 an administrative reform was implemented where some regions were redefined. Consequently 1998 is the first year for which data for the regions in today's structure are available. Therefore we decided to start our analysis with the year 1998 to avoid additional aggregation or disaggregation biases, which might occur in the data before 1998.

In addition we used the data for Uzbekistan as well, because a group of regions with similar steady states is not necessarily defined by country borders. An example for this would be the North and South Italian regions. GRP data on the 14 Uzbek regions are taken from the National Human Development Report 2007. As the GRP data sets for Kazakhstan and Uzbekistan are both in US-Dollar at PPP they can easily be merged.

\section{Theoretical background of convergence analysis}

Generally spoken the idea of convergence is an outcome of the neoclassical growth theory done for example by Solow (1956). Basic framework conditions for such a model are a perfect market and linearity in the aggregated production function (Barro und Sala-i-Martin, 2004). In literature two different concepts are distinguished. The first concept is the so called $\beta$ convergence, which is based on the fact that poor regions tend to grow faster than rich ones and per capita income of the former, would catch up with the latter. The main assumption here is that there is a common steady state across the analyzed countries or regions. The second concept is called $\sigma$-convergence which will be explained in detail in the following paragraph.

\section{1 $\sigma$-convergence}

Following Barro and Sala-i-Martin (2004) $\sigma$-convergence is defined in terms of the level of income dispersion. In principle $\sigma$-convergence prevails if the cross sectional dispersion of the GRP per capita declines over time. In this paper it is measured by the standard deviation of the annual $\log$ of GRP.

\section{$3.2 \beta$-convergence}

Countries or regions with similar technologies and preferences tend to have identical steady states. In this framework poor regions tend to grow faster then rich ones. The theory behind this is known as absolute or unconditional $\beta$-convergence theory. The initially poorer regions tend to grow faster than the initially rich ones due to diminishing returns on capital given a common steady state as the basic underlying assumption. The correlation between the initial level of per capita GRP and its average growth rate is represented by the following equation:

$$
\operatorname{h}\left(\frac{y_{i, t}}{y_{i, t}}\right)\left(\frac{1}{T}\right)=a_{i, t}+b^{*} \operatorname{h}\left(y_{i, E}\right)+u_{i z}
$$

On the left hand side in the above equation we have the average annual growth rate of the per capita GRP. On the right hand side refers to the level of per capita GRP, subscript denotes the year and subscript the region. indicates the length of the observed time period. The error term is distributed independently with mean 0 and variance. Roughly spoken, if the log of the initial GRP per capita is negatively related to the respective average annual growth rate, absolute $\beta$ convergence is said to be present. $\beta$, which is included in $b$ as we will show later can be interpreted as the speed of convergence, which is approximately $2 \%$ according to empirical estimations. 


\section{Empirical results}

\section{1 $\sigma$-convergence}

As indicated above the existence of $\sigma$-convergence implies that the dispersion of per capita product, measured as the standard deviation of per capita GRP across regions, declines over time.

Figure 1 shows the cross-sectional standard deviation (i.e. sigma convergence) of the log of per capita GRP for the period 1998 to 2008. The solid line graphs the movement of $\sigma$ convergence, including all Kazakh regions. The dashed line describes the $\sigma$-convergence within the sub-set of regions, which are well endowed with oil. These are Aktyubinsk, Atyrau, Kyzylorda, Mangistau, and Western Kazakhstan. Regions without oil are represented by the third line.



Figure 1. Standard deviation of the log of per capita GRP across Kazakh regions, 1998-2008

The lines can be interpreted in the following way. As the variation in per capita GRP is growing over time (except in 2001 and 2007), no $\sigma$-convergence across all Kazakh regions seems to be present. As the trend for all regions coincides with the one for the regions without oil, $\sigma$-convergence could also not be established for these regions.

In contrast, there is evidence for $\sigma$-convergence across the oil regions. The variation in per capita GRP in these regions grows steeply during the first two years, but declines steadily in the following years. In 2007 it even goes below the starting level. The reason for this might be that according to Najman et al. (2005) Atyrau and Mangistau have traditionally been the main oil producers whereas Aktyubinsk, Kyzylorda and Western Kazakhstan are three relatively new oil-producing regions, which were only explored during the last decade. That is why the latter caught up with Atyrau and Mangistau between 2000 and 2007 while expanding the oil production gradually.

\subsection{Absolute $\beta$-convergence}

The focus of the $\beta$-convergence is the speed of adjustment. To get this immediately it is necessary to estimate a non-linear equation which is represented by the following equation: 


\section{$\operatorname{h}\left(\frac{y_{i z z}}{y_{i z-1}}\right)\left(\frac{1}{T}\right)=a_{i z}-\left(1-e^{-p}\right) h\left(y_{i z-1}\right)+u_{i z}$}

On the left hand side we have again the average annual growth rate and on the right hand side of the non-linear estimation equation we use the parameter matching those from the theoretical paragraph. Here is the parameter estimating the rate of the technological progress which should decline with a rising time interval. Again the time interval is indicated by $\mathrm{T}$. Hence the weight of the initial growth rate is higher if the time span is shorter.

Estimating the equation like it is presented in the theoretical part with OLS leeds to the same result, but the speed of convergence has then to be calculated separately with the following equation:

$$
b=-\left(\frac{1}{T}\right)\left[1-e^{-\rho r}\right]
$$

The univariate non-linear regression equation is estimated with the above mentioned data for the 16 Kazakh regions for the period 2000-2005. We also did some robustness checks using different time periods and received similar results. To do further checks on the robustness of our results, we added the regions of Uzbekistan and estimated the equation again with 30 observations. The underlying assumption is that the Kazakh and Uzbek regions have the same steady state.

(1)

Kazakhstan
(2)

Kazakhstan \&

Uzbekistan

$$
(-1,14)
$$

\begin{tabular}{ccc} 
Observations & 16 & 30 \\
R-squared & 78 & 265 \\
\hline
\end{tabular}

$$
\text { *** } \mathrm{p}<0.01,{ }^{* *} \mathrm{p}<0.05,{ }^{*} \mathrm{p}<0.1
$$

\section{Table 1. Results of non-linear estimations}

The results of our estimations are shown in table 1 . The beta coefficient has a negative sign but is not statistically significant different from zero. The negative sign would indicate that there is no absolute $\beta$-convergence across Kazakh regions. The beta coefficient of the full sample estimation, including Uzbekistan, is significantly different form zero and again negative. So there seems not to be any evidence for absolute $\beta$-convergence across these regions. This can be interpreted as an evidence for the correctness of our results for Kazakhstan, namely, that there is no regional $\beta$-convergence across Kazakh regions. This outcome coincides with the result of the $\sigma$-convergence.

\section{Conclusion}

In this paper we have shown that there is no evidence for regional $\sigma$ - and absolute $\beta$ convergence in Kazakhstan so far. The only evidence for $\sigma$-convergence can be seen if we look solely at the oil producing regions. This indicates that oil plays an important role in the convergence process, which leads us to the missing concept of conditional $\beta$-convergence, where additional explanatory variables are introduced as proxies for different steady states. As a next step we will have a closer look at the conditional $\beta$-convergence across Kazakh regions. 


\section{References}

- Agrawal, Pradeep, 2008. "Economic Growth and Poverty Reduction: Evidence from Kazakhstan“, Asian Development Review, vol. 24, no. 2, 90-115.

- Barro, Robert J., et al., 1991. "Convergence across States and Regions”, Brooking Papers on Economic Activity, no. 1, 107-182.

- Barro, Robert J., and Sala-i-Martin, Xavier, 1992. "Convergence”, Journal of Political Economy, 100(2):223-251.

- Barro, Robert J., and Sala-i-Martin, Xavier, 2004. Economic Growth. The MIT Press, Cambridge (Massachusetts).

- $\quad$ Najman, Boris et al., 2005. "How are Oil Revenues redistributed in an Oil Economy?", The University of Adelaide, School of Economics, Working Paper 200518.

- O‘Hara, Sarah, and Gentile, Michael, 2009. "Household Incomes in Central Asia: The Case of Post-Soviet Kazakhstan“, Eurasian Geography and Economics, 2009, 50, no. 3, pp. 327-347.

- Solanko, Laura, 2008. "Unequal fortunes: a note on income convergence across Russian regions", Post-Communist Economies, vol. 20, September 2008, 287-301.

- Solow, Robert M., 1956. "A Contribution to the Theory of Economic Growth", Quarterly Journal of Economics, vol. 70, 65-94.

- UNDP, 2007/2008. Education in Uzbekistan: matching supply and demand. Report UNDP Uzbekistan, United Nations Development Programme, Tashkent 2007/2008.

- UNDP, 2009. From exclusion to equality: realising the rights of persons with disabilities in Kazakhstan. Report UNDP Kazakhstan, United Nations Development Programme, Kazakhstan 2009. 